\title{
Deux disques sur les minorités du Viêt-Nam
}

\section{Dana Rappoport}

\section{(2) OpenEdition \\ Journals}

Édition électronique

URL : http://journals.openedition.org/ethnomusicologie/736

ISSN : 2235-7688

Éditeur

ADEM - Ateliers d'ethnomusicologie

Édition imprimée

Date de publication : 1 janvier 2001

Pagination : 247-250

ISBN : 2-8257-0723-6

ISSN : 1662-372X

Référence électronique

Dana Rappoport, «Deux disques sur les minorités du Viêt-Nam », Cahiers d'ethnomusicologie [En ligne], 13 | 2001, mis en ligne le 09 janvier 2012, consulté le 20 avril 2019. URL : http:// journals.openedition.org/ethnomusicologie/736

Ce document a été généré automatiquement le 20 avril 2019.

Tous droits réservés 


\section{Deux disques sur les minorités du Viêt-Nam}

\section{Dana Rappoport}

\section{RÉFÉRENCE}

Viêt-nam. Anthologie de la musique Êdê. Enregistrements, textes et photos (1996-1998) :

Patrick Kersalé, Franck Barthélémy ; traduction anglaise de Dominique Bach. Notice bilingue français/anglais de 31 pages, 10 photos couleurs, 2 planches d'illustrations. Collection Musique du monde, 1 CD Buda Records 92726-2.

Viêt-nam. Musiques et chants des minorités du nord. Enregistrements (1995), textes et photos : Patrick Kersalé. Notice bilingue français/anglais de 28 pages, 9 photos couleurs en recto de couverture. Collection Musique du monde, 1 CD Buda Records 92669-2.

1 Depuis 1993, Patrick Kersalé se rend régulièrement au Viêt-nam : il a déjà réalisé dix disques sur les musiques traditionnelles du Viêt-nam ${ }^{1}$, et un onzième - sur les musiques Joraï - sortira sous peu. La variété et la quantité de ces enregistrements font du catalogue de Kersalé l'un des plus riches concernant le Viêt-nam dans le domaine de l'édition discographique française. Le Viêt-nam ne constitue pourtant pas sa seule aire d'investigation puisque depuis six ans, il a déjà fait paraître 48 disques sur les musiques du monde. Véritable boulimique de musiques traditionnelles, Patrick Kersalé est un homme pressé qui explore le monde avec son DAT sans s'arrêter, dans un élan vertigineux.

2 Rappelons tout d'abord que les minorités du Viêt-nam, souvent classées en minorités du centre et du nord, représentent $15 \%$ de la population totale soit douze millions de personnes parlant 53 langues différentes (en excluant la langue de l'ethnie majoritaire kinh), chacune d'elle relevant d'une des cinq grandes familles linguistiques présentes au Viêt-nam: les familles austro-asiatique (ou mon khmer), austronésienne, thaï-kadaï, miao-yao, tibéto-birmane². 


\section{Viêt-nam. Anthologie de la musique Êdê}

3 Le premier disque est simplement exceptionnel : il nous fait découvrir des organisations musicales stupéfiantes de simplicité complexe. Des hoquets de sifflets (ding tut) comme on en a rarement entendus, voilà ce qui retient l'attention dans ce disque consacré à la musique des Edê (autrefois appelés Rhadé), un peuple situé sur le plateau du Dak Lak et parlant une langue austronésienne. Une étude ethnologique française leur a déjà été consacrée ${ }^{3}$, dans laquelle est décrite leur société matrilinéaire - mais Patrick Kersalé, malheureusement, ne la mentionne pas dans la notice.

4 Les locuteurs de langues austronésiennes sont plus de 270 millions, répartis de Madagascar à l'île de Pâques. La plupart d'entre eux vivent dans les îles (Madagascar, Taïwan, Indonésie, Philippines et de nombreuses îles d'Océanie). Les rares populations austronésiennes de l'Asie du Sud-Est péninsulaire vivent précisément sur les hauts plateaux du Viêt-nam et sur les côtes de Birmanie et de Thaillande. Ainsi, l'utilité des enregistrements de Patrick Kersalé est évidente : en plus de leur qualité esthétique, ils permettront de contribuer à la reconstitution du tableau de la famille musicale austronésienne. C'est donc un document important que nous avons à présent entre les mains.

5 Ce disque donne un très bon aperçu sonore de l'esthétique musicale êdê, encore très homogène tant sur le plan instrumental que vocal: les extraordinaires polyphonies instrumentales de sifflets (plages 1 à 11) présentent les mêmes caractéristiques que les polyphonies vocales (plages 14 et 15) ou que les polyrythmies de bambous percutés (plage 30). Haletantes et rythmées, les pièces sont formées d'un ou deux motifs répétés qui s'inscrivent dans une cellule de quatre temps, utilisant un ambitus limité. La spécificité musicale des musiques êdê réside en effet dans l'emploi d'une technique de hoquet et la répétition sur de courtes cellules de quatre temps, dans une homogénéité et une plénitude uniques. Cette organisation semble à l'œuvre dans la plupart des musiques d'ensemble. Musique très répétitive et surprenante par sa densité. Cette esthétique n'est pas véritablement décrite par le collecteur qui s'attache davantage à décrire la composition des ensembles.

6 Le disque offre une large place aux ensembles de sifflets, aux gongs et tambours, relativement bien décrits dans la notice. Les polyphonies de six sifflets en bambou ding tut (plages 1 à 9), pouvant être quelquefois accompagnées d'une voix soliste (plage 10) fondent, semble-t-il, l'esthétique musicale êdê.

7 D'autres instruments de musique, souvent résiduels, sont aussi présentés : orgue à bouche à six tuyaux ding nam, cithare sur bâton à double cordes bro', tambour à deux peaux h'gor, ensemble de gongs cing, flûte ding buot, guimbarde goc, aérophone ding ring, aérophone à anche libre ding tak ta..

8 Une fois n'est pas coutume, les femmes ont une place musicale importante et singulière puisqu'elles jouent des instruments à vent: sifflets (ding tut), orgue à bouche (ding nam), flûte (ding buot).

9 Les exemples vocaux - chœur (plage 14 et 15), chant accompagné (plage 10), invocation (plage 27), psalmodie (plage 28) sont moins bien représentés.

10 Comparés aux notices d'autres disques de P. Kersalé, la notice du disque Edê a été mieux documentée qu'à l'accoutumée - non seulement dans la description (on y trouve tous les 
noms des instruments et leur organisation) mais aussi dans les planches d'illustrations, très utiles, et bien plus descriptives que des termes d'organologie souvent peu évocateurs pour le lecteur non expert.

11 Il reste pourtant des maladresses dans la présentation ethnographique et littéraire, maladresses trop importantes pour ne pas être soulignées. C'est avec un aplomb intrépide que l'auteur pose une construction anthropologique qu'un ethnologue aurait mis dix ans à dévoiler par une argumentation serrée. On est gêné de voir réglé le sort de cette société en une page de notice (p. 3) : «la société matrilinéaire», «le culte des esprits», «le dualisme et la dichotomie ", "la symbolique des chiffres", tout cela sans la moindre référence. De même, la description de la stylistique êdê relève presque de la parodie scientifique (p. 4-5). On comprend dès lors comment Patrick Kersalé a pu réaliser une telle quantité de disques en si peu de temps : n'est-il pas plus rapide d'aller sur le terrain et de réaliser un disque que de décrire et documenter ces musiques? Tous les ethnomusicologues le savent. Pourquoi ne pas ralentir la course et prendre un peu le temps de nous aider à écouter et à saisir les structures mentales des musiciens? Est-ce que l'urgence dont on parle tant dans notre discipline est à ce point dramatique qu'on ne puisse bien documenter nos enregistrements?

Hormis cette réserve, sur le plan sonore, cet excellent disque présente une véritable unité musicale alliant le plaisir de l'ouïe avec l'exaltation de la connaissance.

\section{Viêt-nam. Musiques et chants des minorités du nord}

Le second disque présente des enregistrements de musiques de huit minorités du nord (Giáy, Muòng, Nùng An, Tày, Dao, Thái, Hmong bariolé, Hmong noir) ainsi que de l'ethnie majoritaire Kinh qui représente $85 \%$ de la population. Le titre ne correspond donc pas précisément au contenu car les Kinh (viêt) ne constituent pas vraiment une minorité. Passons sur le détail.

Les pièces sont présentées par ethnies - en moyenne, une à trois pièces pour chacune, sauf pour les Thái dont on peut entendre cinq exemples. Le disque est composé d'une alternance de pièces pour ensemble, de chants et d'aérophones en solo (orgue à bouche khen, aérophone en bambou pí pãp...). On découvre quatre types de petites formations instrumentales : 1 . hautbois, cymbales, tambour et gongs ; 2 . flûtes et vièle à deux cordes ; 3. tambour suspendu et gongs à mamelon; 4. hautbois, vièle à deux cordes, tambour à deux peaux. La classification des chants reste sommaire; le collecteur persiste dans l'utilisation de catégories occidentales : «chant d'accueil », «chant d'amour ", « chant à caractère champêtre »....

La musique séduit l'oreille par la grande beauté de certaines pièces (et notamment des pièces chantées - plages $6,9,15)$. Le tout est très bien enregistré - et il faut à nouveau saluer l'exploit de nous faire découvrir tant de formes différentes quand on sait la difficulté de la recherche au Viêt-nam. La variété d'exemples enrichira la documentation sonore.

Pourtant, une fois encore, la notice laisse vraiment à désirer. N'est-il pas à regretter que toute description du contexte soit laissée de côté ? Peut-on valoriser la variété musicale sans une pensée analytique permettant une finesse descriptive alliée à une largeur de synthèse? Comment prétendre saisir l'unité, l'identité d'une culture par un ou deux 
exemples sonores inégalement documentés? Un collecteur peut-il se contenter d'enregistrer sans documenter correctement les musiques?

Le but de Patrick Kersalé est clair : donner à écouter des musiques qui disparaissent, sans les analyser. Il laisse ce travail à d'autres. Mais qui le fera ? Faut-il surmonter à nouveau tous les obstacles qui nous obstruent la route vers le terrain? Malgré ses talents de musicien, pourquoi le collecteur ne prend-il pas la peine d'attacher davantage de soin au détail ? A force de variété et de quantité, il en oublie la précision de la documentation. Le manque de soin apporté à la notice entraîne également de légères fautes d'analyse musicale tel ce chant de la plage 15 présenté par sa "gamme tétratonique » alors qu'il se déploie sur une échelle pentatonique anhémitonique. Détail, mais à force d'imprécisions, on se met à douter.

Patrick Kersalé est un curieux paradoxe : il bouge vite, il produit plus rapidement que quiconque et donne à entendre des formes sonores toujours nouvelles, souvent extraordinaires, bien enregistrées, mais il ne prend pas la peine de les présenter avec prudence et attention. Or n'est-ce pas le but de notre travail de passeurs, que de donner aux auditeurs des clés d'écoute?

BIBLIOGRAPHIE

\section{Précédents enregistrements de Patrick Kersalé sur les musiques du Viêt-nam}

Anthologie de la musique êdê. Coll. Musique du monde. 1 CD Buda Musique 92726-2.

Chants de possession. Coll. Musique du monde. 1 CD Buda Musique 92657-2.

Chants des minorités des hauts plateaux du nord. Coll. Peoples. 1 CD VDE-Gallo 826.

L'art de la vièle vietnamienne. Coll. L'art de... 1 CD Arion ARN 60417.

L'art du khèn Coll. L'art de... 1 CD Arion ARN 60367.

Musique et chants des Hmong. Coll. Peoples. 1 CD VDE-Gallo 915.

Musique funéraire du nord. Coll. Musica Deo. 1 CD Arion ARN 58456.

Musiques et chants des minorités du Nord. Coll. Musique du monde. 1 CD Buda Musique 92669-2.

Théâtre populaire du nord (Hát chèo). 1 CD Arion ARN 64368.

Vietnam, une tradition rénovée. Coll. Playa Sound. 1 CD Sunset France PS 65116.

\section{NOTES}

1. Voir liste en annexe. 
2. Cf. la notice du récent coffret Vietnam, Musiques des montagnards. Collection CNRS / Musée de l'Homme. 2 CD Le Chant du Monde CNR 2741085.86.

3. Anne de Hauteclocque-Howe: Les Rhadés une société de droit maternel. Editions du CNRS, 1985. 